\title{
Transcarotid access for remote robotic endovascular neurointerventions: a cadaveric proof-of-concept study
}

\author{
*Marton Berczeli, MD,,2 Ponraj Chinnadurai, MBBS, MMST,1,3 Peter T. Legeza, MD,,2 \\ Gavin W. Britz, MD, MPH, MBA, ${ }^{4}$ and Alan B. Lumsden, MD ${ }^{1}$ \\ 1Department of Cardiovascular Surgery, Houston Methodist Hospital, Houston; ${ }^{4}$ Department of Neurological Surgery and \\ Neurological Institute, Houston Methodist Hospital, Houston, Texas; '2Department of Vascular and Endovascular Surgery,

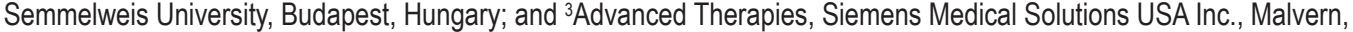 \\ Pennsylvania
}

OBJECTIVE The purpose of this proof-of-concept study was to demonstrate the setup and feasibility of transcarotid access for remote robotic neurointerventions in a cadaveric model.

METHODS The interventional procedures were performed in a fresh-frozen cadaveric model using an endovascular robotic system and a robotic angiography imaging system. A prototype remote, robotic-drive system with an ethernetbased network connectivity and audio-video communication system was used to drive the robotic system remotely. After surgical exposure of the common carotid artery in a cadaveric model, an 8-Fr arterial was inserted and anchored. A telescopic guiding sheath and catheter/microcatheter combination was modified to account for the "workable" length with the CorPath GRX robotic system using transcarotid access.

RESULTS To simulate a carotid stenting procedure, a 0.014-inch wire was advanced robotically to the extracranial internal carotid artery. After confirming the wire position and anatomy by angiography, a self-expandable rapid exchange nitinol stent was loaded into the robotic cassette, advanced, and then deployed robotically across the carotid bifurcation. To simulate an endovascular stroke recanalization procedure, a 0.014-inch wire was advanced into the proximal middle cerebral artery with robotic assistance. A modified 2.95-Fr delivery microcatheter (Velocity, Penumbra Inc.) was loaded into the robotic cassette and positioned. After robotic retraction of the wire, it was switched manually to a mechanical thrombectomy device (Solitaire X, Medtronic). The stentriever was then advanced robotically into the end of the microcatheter. After robotic unfolding and short microcatheter retraction, the microcatheter was manually removed and the stent retriever was extracted using robotic assistance. During intravascular navigation, the device position was guided by 2D angiography and confirmed by 3D cone-beam CT angiography.

CONCLUSIONS In this proof-of-concept cadaver study, the authors demonstrated the setup and technical feasibility of transcarotid access for remote robot-assisted neurointerventions such as carotid artery stenting and mechanical thrombectomy. Using transcarotid access, catheter length modifications were necessary to achieve "working length" compatibility with the current-generation CorPath GRX robotic system. While further improvements in dedicated robotic solutions for neurointerventions and next-generation thrombectomy devices are necessary, the transcarotid approach provides a direct, relatively rapid access route to the brain for delivering remote stroke treatment.

https://thejns.org/doi/abs/10.3171/2021.10.FOCUS21511

KEYWORDS trans-carotid approach; direct carotid access; endovascular robot; remote interventions; remote neurointervention; remote stroke intervention; remote mechanical thrombectomy

$\mathrm{E}$ NDOVASCULAR robotics has shown promise in the field of interventional cardiology and vascular surgery. ${ }^{1-3}$ The advantages of robotic navigation include fine dexterity leading to precise wire navigation and device positioning, and lower radiation exposure, especially for the primary interventionalist or surgeon..$^{1-3}$ With advances in remote technologies and network connectivity, robot-assisted interventions can be performed remotely, democratizing expert care and healthcare delivery. This has recently been demonstrated in telerobotic percutaneous coronary intervention (PCI), where the expert interventionalist was delivering therapy approximately 20 miles away from the patient. ${ }^{4}$ Future possibilities of such remote robot-assisted procedures have led to increased

ABBREVIATIONS MT = mechanical thrombectomy; $\mathrm{PCI}=$ percutaneous coronary intervention; $\mathrm{TCAR}=$ transcarotid artery revascularization

SUBMITTED September 1, 2021. ACCEPTED October 22, 2021.

INCLUDE WHEN CITING DOI: 10.3171/2021.10.FOCUS21511.

${ }^{*}$ G.W.B. and A.B.L. contributed equally to this work and share senior authorship. 
TABLE 1. Endovascular devices used for the transcarotid approach

\begin{tabular}{|c|c|c|c|}
\hline Product Name & Size & Length & Company \\
\hline Runthrough NS wire & 0.014 inch & $300 \mathrm{~cm}$ & Terumo \\
\hline Enroute Transcarotid Stent System & $8 \mathrm{Fr}$ & & Silk Road Medical \\
\hline Pinnacle Destination straight guiding sheath & $6 \mathrm{Fr}$ & $\begin{array}{l}45 \mathrm{~cm} \& \text { physician-modified shorter to } \\
\text { connect to TCAR sheath system }\end{array}$ & Terumo \\
\hline Mach 1 guiding catheter & $6 \mathrm{Fr}$ & $\begin{array}{l}\text { Cut to } 15 \mathrm{~cm} \text { to connect to TCAR sheath } \\
\text { system }\end{array}$ & Boston Scientific \\
\hline Carotid Wallstent & $\begin{array}{l}\text { 6-Fr guiding sheath \& guiding } \\
\text { catheter compatible }\end{array}$ & $135 \mathrm{~cm}$ & Boston Scientific \\
\hline Velocity delivery microcatheter, straight tip & $2.95 \mathrm{Fr}$ & $160 \mathrm{~cm}$ & Penumbra Inc. \\
\hline Solitaire X 4-mm $\times 20$-mm revascularization device & $4 \mathrm{~mm}$ & $200 \mathrm{~cm}$ & Medtronic \\
\hline
\end{tabular}

interest from the interventional neuroradiology and neurosurgical community. However, neurovascular interventions often require a triaxial system with need for microcatheter support. Recently, the CorPath GRX (Corindus Inc.) platform has been adapted to handle microcatheters and active device fixation, and, thereby, facilitate neurovascular interventions. ${ }^{5,6}$ The current-generation CorPath GRX system has recently received the European conformity (CE) mark for neurovascular interventions, although it is FDA approved only for PCI and peripheral vascular interventions.

Stroke poses a significant burden on our healthcare system and is estimated to affect approximately $4 \%$ of the US population by $2030 .^{7}$ Mechanical thrombectomy (MT) is the standard-of-care therapy for large-vessel occlusions, ${ }^{8}$ but the timing of interventional treatment dictates the clinical outcome. ${ }^{9}$ Remote, robotic, interventional stroke treatment could be a potential solution to delivering therapies such as MT by expert neurovascular specialists without transporting patients hundreds of miles to a tertiary care center. Although femoral arterial access is the routinely performed standard, it can be technically challenging in select patients due to anatomical factors such as high bifurcation of the common femoral artery or tortuous iliac artery, or heavily calcified/stenotic vessels. The subsequent interventional step of cannulating and navigating arch vessels may be difficult in patients with challenging arch anatomy such as bovine arch, type III arch, rightsided arch, and in those who have received prior stents or debranching surgery. In the absence of distal-articulating, steerable catheters with tip localization in 3D space, the task of manually navigating long catheters and wires inside the brain from at least 3 feet away (femoral approach) using 2D fluoroscopy is cumbersome due to the buildup of mechanical forces such as torque and friction. The challenges associated with femoral access and aortic arch cannulation may lead to delayed or even aborted MT, both of which may be detrimental, especially in the setting of acute ischemic stroke. Recent studies have shown the safety and efficacy of alternate nonfemoral access strategies, such as the transcarotid approach, for MT. ${ }^{10-12}$

Our experience with the transcarotid artery revascularization (TCAR) procedure, and the favorable results it delivers with flow reversal compared with conventional carotid artery stenting, ${ }^{13}$ has provided us further impetus to explore robot-assisted neurovascular interventions via the direct transcarotid approach in the setting of acute ischemic anterior circulation stroke.

The primary objective of this proof-of-concept study was to demonstrate the technical feasibility of transcarotid access as an alternative to transfemoral access, in performing robot-assisted carotid artery stenting and endovascular MT in a cadaveric model.

\section{Methods}

\section{Transcarotid Access and Imaging Setup}

The procedure was performed on a fresh-frozen cadaver in a hybrid operating room equipped with a robotic angiography imaging system (Artis zeego, Siemens Healthineers AG) in a preclinical, surgical-training facility. Prior to any interventions, arterial and venous thrombectomy was performed using a suction thrombectomy system (Indigo Aspiration System, Penumbra Inc.). Bilateral common femoral veins were exposed by femoral cutdown. Then, the 8-Fr venous sheaths of the Enroute TranscarotidStent System (Silk Road Medical) were inserted. For the transcarotid approach, bilateral common carotid arteries were exposed using a transverse surgical incision above the clavicle. Cervical common carotid arteries were controlled with an umbilical tape proximally and with vessel loops distally, then a small transverse arteriotomy was performed. An 8-Fr arterial sheath of the Enroute stent system was inserted into the bilateral common carotid arteries and sutured to the skin. To facilitate robot-assisted endovascular neurointerventions, 2 short, straight 6-Fr guiding sheaths (Pinnacle Destination guiding sheath, Terumo [1 standard, 1 modified] and also a modified 6-Fr Mach 1 guiding catheter) were used. Table 1 summarizes the devices used during the study. Intraoperative 2D angiography and cone-beam CT angiography (syngo DynaCT, Siemens) were performed to assess vascular anatomy and ascertain accurate device positioning after manual injection of the iodinated contrast agent and saline flushes throughout the experiment.

\section{Local and Remote Robotic Setup}

To test the feasibility of the transcarotid access for remote, robot-assisted neurointerventions, the CorPath GRX endovascular robotic system, equipped with prototype 


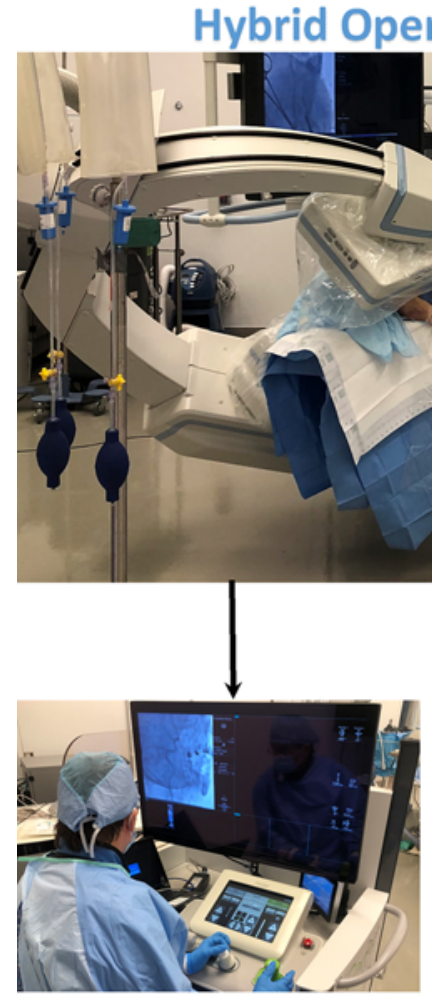

Local Console xperimental setup. For local robot-assisted
perator sits behind the radiation-shielded intervention, the primary operator sits behind the radiation-shielded cockpit inside the hybrid suite. For the remote procedure, the primary operator is outside the hybrid suite; the local and remote sites are synchronized with a computer, and fluoroscopic images are displayed on its monitor. An additional audio-visual connection is established between the local and remote teams.

telerobotic functionality, was used. The robotic system setup was described in detail previously. ${ }^{14,15}$ The robotic drive was mounted onto the hybrid operating room table, then a robotic cassette was connected to the robotic arm and positioned above the chest and abdomen of the cadaver. The robotic cassette is compatible with a 0.014 -inch wire and monorail/rapid exchange devices. Figure 1 illustrates the setup and control for both local and remote robotic systems. The local console was connected to the main robotic drive with a cable. The remote console was connected via local ethernet to a telerobotic system equipped with a remote robotic control, monitors, and bidirectional audio-video communication. An institutional network was used during the whole procedure with a mean \pm SD latency time between remote joystick movement and actual robotic movement of $55.4 \pm 7.5 \mathrm{msec}$, with a 0 -msec image delay.

\section{Results \\ Robotic Carotid Stenting Using Transcarotid Access}

After surgical exposure of the common carotid artery, an 8-Fr TCAR sheath was inserted, as described above. To connect the robotic cassette to the TCAR sheath, a 45cm-long, straight 6-Fr guiding sheath (Pinnacle Destination guiding sheath, Terumo) and a 6-Fr guiding catheter were cut to a shorter length and inserted through the hemostatic valve of the arterial TCAR sheath, as illustrated in Fig. 2. To ensure flow reversal during the procedure, the modified guiding sheath should not cover the side-port of the arterial stopcock that connects with the femoral venous sheath. The distal end of the guiding sheath/catheter was connected to an adapter and a Y-connector that connects to the robotic cassette, then the cassette was secured to the hemostatic valve of the TCAR arterial sheath using the guide catheter track. After connecting the robotic cassette, a 300-cm-long, 0.014-inch floppy wire (Runthrough NS wire, Terumo) was advanced, with robotic assistance, into the distal internal carotid artery (Video 1).

VIDEO 1. Robot-assisted carotid stent positioning with the CorPath GRX system using the TCAR sheath system. Copyright Marton Berczeli. Published with permission. Click here to view.

Based on intraoperative angiographic measurements, a 6-Fr guiding sheath compatible, monorail self-expandable
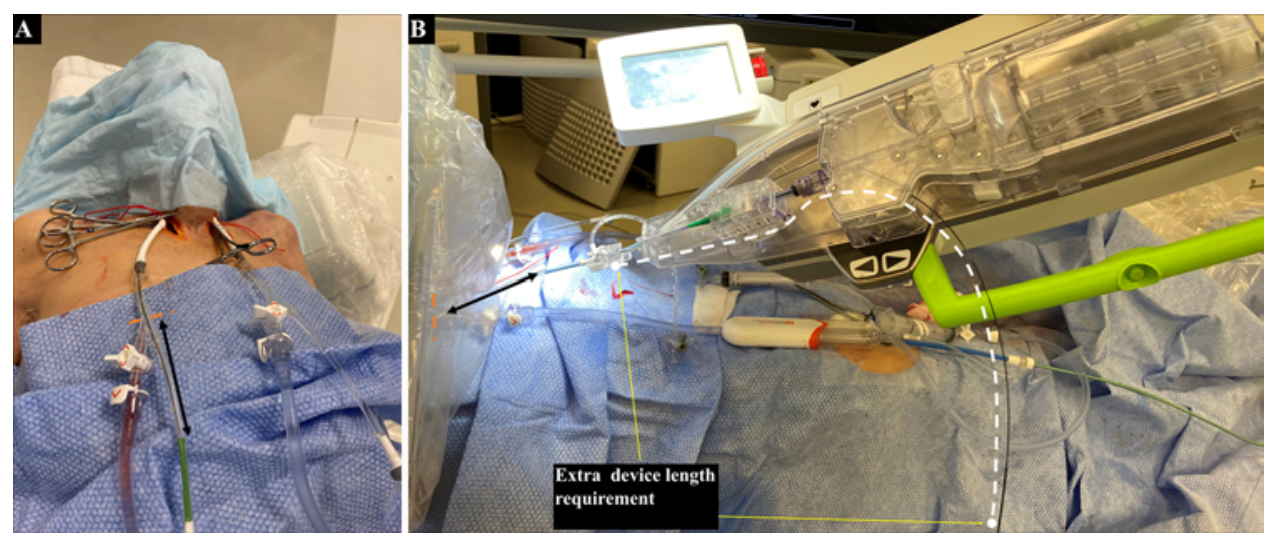

FIG. 2. Transcarotid access with the TCAR sheath system and the connected CorPath GRX system. A: Photograph demonstrating the cut Destination guiding sheath inside the arterial sheath of the TCAR system. The black double-headed arrow indicates the remaining length until blocking the retrograde flow at the orifice of the arterial Y-connector (orange dashed line). B: Photograph illustrating the setup for robot-assisted transcarotid artery stenting. A 6-Fr guiding catheter was used to connect to the sheath system and a monorail self-expandable stent. The original Enroute stent system comes with a $90-\mathrm{cm}$-long catheter; however, the white dashed line illustrates the additional catheter length that the robotic assistance requires, hence, we used a different type of stent. 


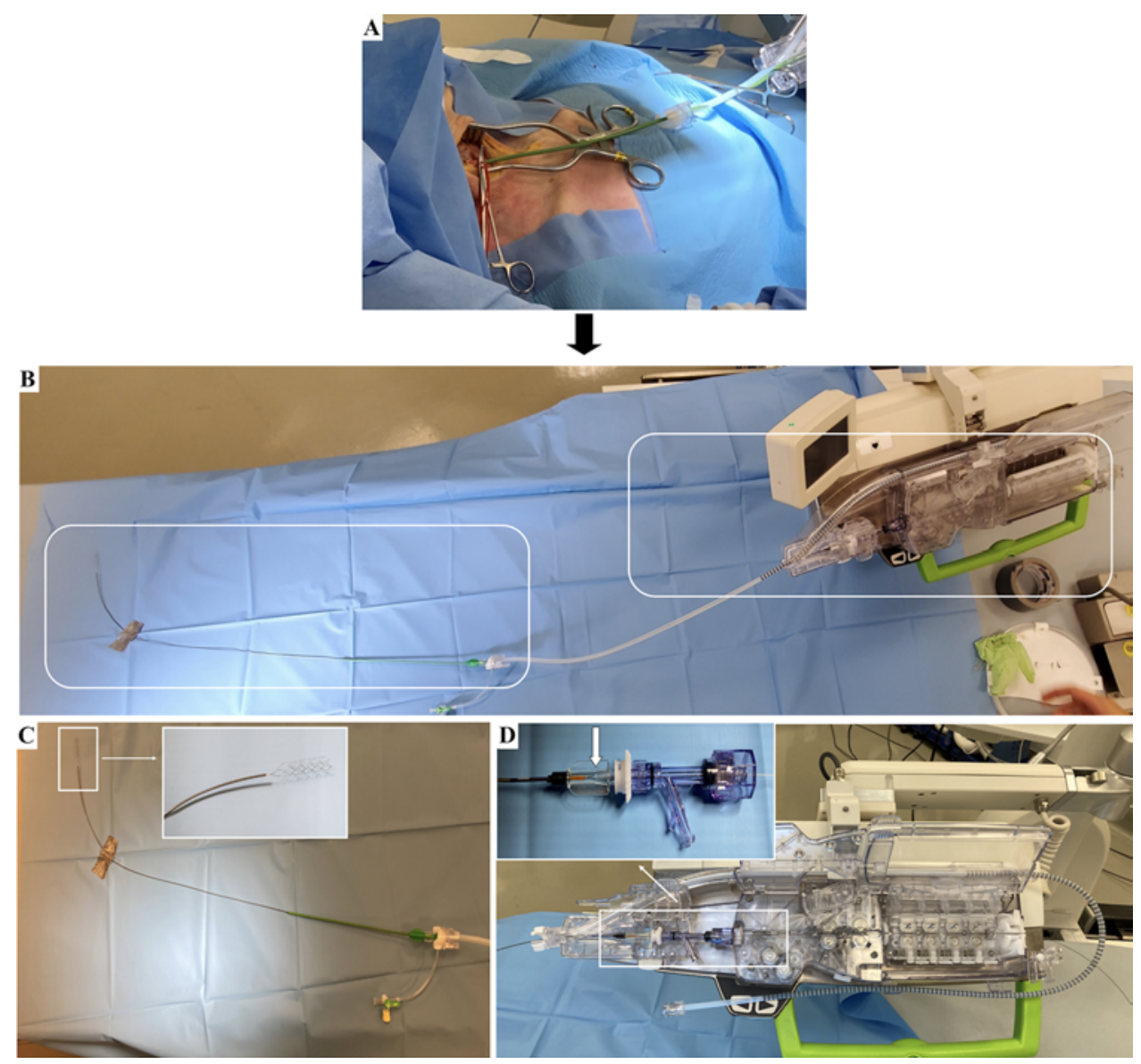

FIG. 3. Simulated intraoperative illustrations of the robotic technical details for transcarotid, robot-assisted endovascular thrombectomy. A: Photograph showing the transcarotid access. B: Photograph showing the general setup of the CorPath GRX system, Velocity microcatheter, Destination guiding sheath, and Solitaire revascularization device. C: Photograph showing the distal tip of the guiding sheath connected to the robotic track; the revascularization device is shown as an inset image at the end of the microcatheter. D: Photograph showing the robotic cassette; the inset image highlights the distal end of the microcatheter (yellow arrow) that is connected to the robotic adapter and the Y-connector.

carotid stent (Carotid Wallstent Monorail Endoprosthesis, Boston Scientific) was loaded onto the robotic cassette. The stent was positioned across the carotid bifurcation with robotic assistance (Video 1), and then was deployed manually. After stent positioning, the delivery system can be placed on a Sonnenburg instrument table to facilitate stable deployment.

\section{Robotic MT Using Transcarotid Access}

After surgical exposure of the common carotid artery, a short, 6-Fr guiding sheath (Destination, Terumo) was inserted to assess feasibility of transcarotid robot-assisted MT using a stentriever. The robotic cassette was connected to the sheath and a 300-cm-long, floppy, 0.014-inch wire (Runthrough) was advanced into the terminal internal carotid artery with robotic assistance. For the procedure, a straight, $160-\mathrm{cm}$ microcatheter was used (Velocity microcatheter, Penumbra Inc.); however, to achieve compatibility between the available $200-\mathrm{cm}$ push-wire length of the stentriever (Solitaire X, Medtronic, $4 \times 20 \mathrm{~mm}$ ) and the robotic system, the proximal end of the microcatheter was cut approximately $20 \mathrm{~cm}$ shorter. Then the wire was manually fed into the microcatheter (Velocity). The distal end of the microcatheter was connected to the robotic cassette, whereby, further advancement of the microcatheter was achieved with robotic assistance. Then the wire was switched to the revascularization device. The stent retriever was loaded onto the distal end of the microcatheter and fed through the Y-connector. This was connected to the cassette, and the stent retriever was fed as a wire. Figure 3 illustrates loading of the stent retriever onto the robotic cassette. An angiography was performed to confirm distal positioning of the microcatheter. The stentriever was then advanced using robotic assistance until reaching the distal end of the microcatheter. The microcatheter was first retracted using robotic assistance (approximately $3 \mathrm{~cm}$ ) to unfold the stent, then disconnected from the robotic cassette and manually retracted. Then the thrombectomy device was reconnected to the cassette and guiding sheath (Destination), and retrieved using robotic assistance. (Video 2).

VIDEO 2. Robot-assisted remote retrieval of the stentriever device (Solitaire X). Copyright Marton Berczeli. Published with permission. Click here to view. 

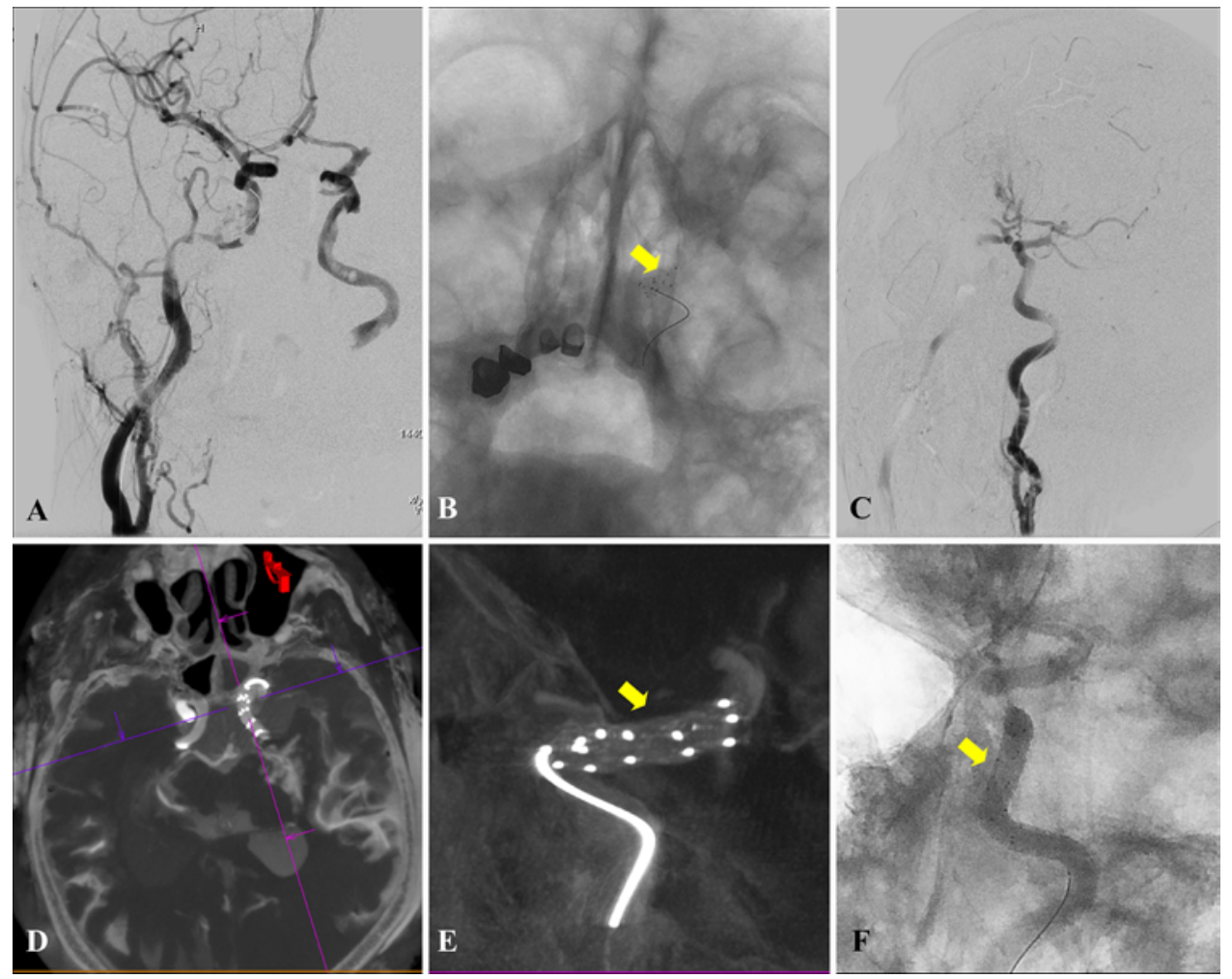

FIG. 4. Imaging during robotic stroke intervention in a cadaveric model. A: Digital subtraction angiogram showing opacification of the right internal and external carotid arteries and partial cross-filling of the left internal carotid artery. B: Frontal plane cine MR image showing the stentriever (Solitaire X) positioned in the left internal carotid artery (yellow arrow). C: Left internal carotid angiogram during mechanical recanalization using the stentriever. D: Axial view cone-beam CT image confirming the position of the stentriever in the cavernous left internal carotid artery. E: Coronal view cone-beam CT image confirming the deployment of the stentriever in the distal left internal carotid artery (yellow arrow). F: Fluoroscopic image showing the radiopaque markers of the stentriever during retrieval in the proximal internal carotid artery (yellow arrow).

Figure 4 illustrates the 2D angiographic images and 3D cone-beam CT images of the stentriever during MT in the cadaver model. This procedure was performed using both local and remote consoles.

\section{Discussion}

In this cadaveric study, we have demonstrated the setup and technical feasibility of remote, robot-assisted neurointerventional procedures such as carotid artery stenting and MT using direct transcarotid access. Direct transcarotid access is a viable, straightforward, rapid alternative to transfemoral access for endovascular robot-assisted neurosurgicalinterventions, especially in the setting of acute stroke, as shown in various pilot clinical studies.11,12 The advantages of transcarotid access include relatively quick, predictable surgical exposure achieved by an available, trained surgeon and safe, direct hemostatic control of the common carotid artery, without any difficulties or complications associated with standard femoral access. A recent study has shown that a small subset $(10 \%)$ of patients with acute ischemic stroke who underwent MT after percutaneous transcarotid access had higher recanalization rates and improved functional outcomes compared with that of patients who underwent aborted MT after failed transfemoral access. ${ }^{10}$ Despite its advantages, transcarotid access has not been adopted routinely in clinical practice, due to concerns of iatrogenic dissection, hematoma after sheath removal in the setting of antithrombotic or thrombolytic medications, and nonavailability of dedicated closure devices. ${ }^{10-12}$ The combination of transcarotid access with robotic assistance augments each of their individual benefits, setting up a better access strategy for remote stroke interventions.

The current-generation CorPath GRX robotic system requires rapid-exchange devices with longer delivery to accommodate the additional distance associated with the robotic setup. The advantage of a robot-assisted TCAR carotid stent is that it combines the benefits of using retrograde flow with precise robotic stent positioning while reducing radiation exposure of the interventionalist.

The major clinical need for remote, robotic assistance in neurointerventions is acute stroke treatment using mechanical or aspirational thrombectomy. Currently the CorPath GRX system is not able to perform fully robotassisted remote MT; however, we believe our proof-ofconcept cadaveric study will be a contribution to urge the field in this direction in the near future. Most catheters and delivery systems are designed and tailored for a transfemoral approach, given that the transcarotid approach is not used routinely. Modifying microcatheters by cutting the proximal end of the catheter results in loss of the most 
flexible segment and affects trackability and navigation capabilities. To adapt the transcarotid robotic approach for MT, shorter microcatheters or longer stentrievers are needed. Singer et al. recently published a technical feasibility study for remote robotic MT in an ex vivo phantom model..$^{16}$ We believe that using a fresh-frozen cadaver model for feasibility testing enables a more realistic setup, provides feedback on device-vessel wall interactions, and allows for identification of any pitfalls for intracranial robotic navigation. Navigating long catheters and devices in the brain using the standard femoral access approach sometimes builds up torque in tortuous aortic arch or iliac vessels. Attempts to measure this force in patient-specific, 3D-printed phantoms using force sensors for various distal access neurocatheters have been published previously. ${ }^{17}$ Direct access to the brain via the transcarotid approach, in principle, would facilitate one-to-one translation of robotic movements from the robot to the distal catheter/wire tip, without any additional drag or torque associated with navigating tortuous vessels.

In this remote, robotic, neurointervention feasibility study, none of the interventionalists experienced any perceptible lag, having an approximately 55 msec latency time difference between remote joystick movement and actual robotic movement. Other important aspects to consider for future platforms include improved quality of audio communication using multiuser, wireless headsets and a standardized communication framework during remote procedures. These findings correlate with recent publications on remote interventions, ${ }^{15,18}$ although there are multiple challenges to overcome in the field of remote stroke treatment. ${ }^{17,19,20}$ From a technical standpoint, a triaxial or quad-axial system (guiding sheath, catheter, microcatheter, and wire/stentriever) is needed to achieve fully robotic MT, and longer rapid-exchange devices are needed to facilitate aspiration thrombectomy.

Although this is a proof-of-concept technical feasibility study in a cadaveric model, it brings a few key concepts together-remote surgery, robotic intervention, and direct carotid access-in order to expand the benefits of MT to more patients with acute ischemic stroke. While we recognize that this is an early step in the journey toward fully remote, robotic stroke intervention, several technical, logistical, and legal challenges need to be addressed in the near future. The CorPath GRX system is currently CE marked but not FDA approved for neurointerventional procedures.

Based on our clinical experience with TCAR and remote robotic interventions, we propose transcarotid access in an effort to compartmentalize the challenges associated with remote, robotic stroke intervention. The road to reach the anterior circulation for MT involves four broad interventional steps: femoral arterial access, arch navigation, and extracranial and intracranial navigation. Transcarotid access simplifies the remote MT task by enabling direct and faster access to the brain, thereby addressing the most important steps of intracranial navigation and thrombectomy. As a next step, we intend to perform a robot-assisted, elective TCAR procedure with flow-reversal as a bridge toward future remote, robotic acute stroke intervention. Considering the lack of resources and endovascular skillsets in a remote, robotic stroke setup, the direct carotid ap- proach simplifies the need to have a trained surgeon who is familiar with surgical carotid exposure and basic skills associated with safe carotid sheath insertion and removal. In due recognition of the need for complex postinterventional medical management, patients can be transferred to the nearest hospital equipped with a neurointensive care unit or managed using a robust tele-ICU setup. While the management of procedural complications as well as the financial and legal implications associated with remote, robotic interventions are still nebulous, we believe that there may be an obvious financial benefit in a life-saving treatment that improves functional neurological outcomes in patients who have experienced acute stroke.

\section{Conclusions}

This cadaveric proof-of-concept study has demonstrated the technical feasibility of remote, robot-assisted neurointerventions using transcarotid access. The direct transcarotid approach provides a relatively rapid, straightforward access to neurovasculature for delivering remote stroke treatment such as MT. The current-generation CorPath GRX system is compatible with the TCAR delivery system to perform carotid artery stenting with flow-reversal, while a combination of manual and robotic approaches is needed for MT. For transcarotid access, customized catheter length modifications were necessary to achieve "working length" compatibility with the current-generation CorPath GRX system.

\section{Acknowledgments}

We express our appreciation for preparing the study to Evan Honeycutt, Rebecca Barnes, Tim Edwards, Johnny Tomas, and the Corindus remote support team. We thank the contribution of Mark Trevino (thrombectomy system) and Michael Bryant (TCAR stent system) for providing technical support.

Dr. Berczeli is supported by Semmelweis University's scholarship: "Kiegészítô Kutatási Kiválósági Ösztöndíj” EFOP-3.6.3VEKOP-16-2017-00009.

\section{References}

1. Weisz G, Metzger DC, Caputo RP, Delgado JA, Marshall JJ, Vetrovec GW, et al. Safety and feasibility of robotic percutaneous coronary intervention: PRECISE (Percutaneous Robotically-Enhanced Coronary Intervention) Study. J Am Coll Cardiol. 2013;61(15):1596-1600.

2. Mahmud E, Naghi J, Ang L, Harrison J, Behnamfar O, Pourdjabbar A, et al. Demonstration of the safety and feasibility of robotically assisted percutaneous coronary intervention in complex coronary lesions: results of the CORA-PCI study (Complex Robotically Assisted Percutaneous Coronary Intervention). JACC Cardiovasc Interv. 2017;10(13):13201327.

3. Mahmud E, Schmid F, Kalmar P, Deutschmann H, Hafner F, Rief P, Brodmann M. Feasibility and safety of robotic peripheral vascular interventions: results of the RAPID trial. JACC Cardiovasc Interv. 2016;9(19):2058-2064.

4. Patel TM, Shah SC, Pancholy SB. Long distance tele-roboticassisted percutaneous coronary intervention: a report of firstin-human experience. EClinicalMedicine. 2019;14:53-58.

5. Britz GW, Panesar SS, Falb P, Tomas J, Desai V, Lumsden A. Neuroendovascular-specific engineering modifications to the CorPath GRX Robotic System. J Neurosurg. 2020;133(6): 1830-1836. 
6. Britz GW, Tomas J, Lumsden A. Feasibility of robotic-assisted neurovascular interventions: initial experience in flow model and porcine model. Neurosurgery. 2020;86(2):309-314.

7. Ovbiagele B, Goldstein LB, Higashida RT, Howard VJ, Johnston SC, Khavjou OA, et al. Forecasting the future of stroke in the United States: a policy statement from the American Heart Association and American Stroke Association. Stroke. 2013;44(8):2361-2375.

8. Campbell BCV, Donnan GA, Lees KR, Hacke W, Khatri P, Hill MD, et al. Endovascular stent thrombectomy: the new standard of care for large vessel ischaemic stroke. Lancet Neurol. 2015;14(8):846-854.

9. He AH, Churilov L, Mitchell PJ, Dowling RJ, Yan B. Every 15-min delay in recanalization by intra-arterial therapy in acute ischemic stroke increases risk of poor outcome. Int $J$ Stroke. 2015;10(7):1062-1067.

10. Cord BJ, Kodali S, Strander S, Silverman A, Wang A, Chouairi F, et al. Direct carotid puncture for mechanical thrombectomy in acute ischemic stroke patients with prohibitive vascular access. J Neurosurg. 2020;135(1):53-63.

11. Mokin M, Snyder KV, Levy EI, Hopkins LN, Siddiqui AH. Direct carotid artery puncture access for endovascular treatment of acute ischemic stroke: technical aspects, advantages, and limitations. J Neurointerv Surg. 2015;7(2):108-113.

12. Roche A, Griffin E, Looby S, Brennan P, O'Hare A, Thornton J, et al. Direct carotid puncture for endovascular thrombectomy in acute ischemic stroke. J Neurointerv Surg. 2019; 11(7):647-652.

13. Malas MB, Dakour-Aridi H, Wang GJ, Kashyap VS, Motaganahalli RL, Eldrup-Jorgensen J, et al. Transcarotid artery revascularization versus transfemoral carotid artery stenting in the Society for Vascular Surgery Vascular Quality Initiative. J Vasc Surg. 2019;69(1):92-103.e2.

14. Legeza P, Britz GW, Loh T, Lumsden A. Current utilization and future directions of robotic-assisted endovascular surgery. Expert Rev Med Devices. 2020;17(9):919-927.

15. Legeza P, Britz GW, Shah A, Sconzert K, Sungur JM, Chinnadurai $\mathrm{P}$, et al. Impact of network performance on remote robotic-assisted endovascular interventions in porcine model. J Robot Surg. Published online February 7, 2021. doi: 10.1007/s11701-021-01196-6

16. Singer J, VanOosterhout S, Madder R. Remote robotic endovascular thrombectomy for acute ischaemic stroke. $B M J$ Neurol Open. 2021;3(1):e000141.

17. Mokin M, Waqas M, Setlur Nagesh SV, Karkhanis NV, Levy EI, Ionita CN, Siddiqui AH. Assessment of distal access catheter performance during neuroendovascular procedures: measuring force in three-dimensional patient specific phantoms. J Neurointerv Surg. 2019;11(6):619-622.

18. Legeza P, Sconzert K, Sungur JM, Loh TM, Britz G, Lumsden A. Preclinical study testing feasibility and technical requirements for successful telerobotic long distance peripheral vascular intervention. Int J Med Robot. 2021;17(3):e2249.

19. Berczeli M, Britz GW, Loh T, Lumsden AB. Tele-robotic endovascular interventions and a future prospect in cerebrovascular treatment. Tex Heart Inst J. In press.

20. Panesar SS, Volpi JJ, Lumsden A, Desai V, Kleiman NS, Sample TL, et al. Telerobotic stroke intervention: a novel solution to the care dissemination dilemma. J Neurosurg. 2020; 132(3):971-978.

\section{Disclosures}

Dr. Chinnadurai: senior staff scientist at Siemens Medical Solutions USA Inc. Dr. Lumsden: research support from Siemens Medical Solutions USA Inc.

\section{Author Contributions}

Conception and design: Berczeli, Chinnadurai, Britz, Lumsden. Acquisition of data: all authors. Analysis and interpretation of data: all authors. Drafting the article: Berczeli, Chinnadurai. Critically revising the article: Chinnadurai, Legeza, Britz, Lumsden. Reviewed submitted version of manuscript: all authors. Approved the final version of the manuscript on behalf of all authors: Berczeli. Administrative/technical/material support: Berczeli, Britz, Lumsden.

\section{Supplemental Information \\ Videos}

Video 1. https://vimeo.com/639991458.

Video 2. https://vimeo.com/639994551.

\section{Correspondence}

Marton Berczeli: Houston Methodist Hospital, Houston, TX. marton.berczeli@gmail.com. 